Its moments are easily calculated, thus

$$
\begin{aligned}
\mathscr{E} P_{n} & =(1-s) \sum_{0}^{n-1}(s / 2)^{i}+(s / 2)^{n} \\
& =\frac{1-s+(s / 2)^{n+1}}{1-s / 2}
\end{aligned}
$$

in agreement with the value of $F_{n}$ given in the reference; also

$$
\mathscr{E} P_{n}^{\mathbf{2}}=\frac{1-s+3(s / 4)^{n+1}}{1-s / 4} \quad \text { a.s.o. }
$$

As $n$ tends to infinity one gets the stationary distribution of $P$ values

$$
W i=(1-s) s i
$$

having mean and variance

$$
\begin{aligned}
\mathscr{E} P & =2 \frac{1-s}{2-s} \\
\operatorname{Var} P & =\frac{4 s(1-s)}{(4-s)(2-s)^{2}} .
\end{aligned}
$$

The same formulae apply to a mixture of random and parent-offspring mating in the case of sex-linked traits or haplo-diploidy, as in bees, provided that "haploid" generations are not counted. More precisely, if $n$ is even, $W_{i}^{(n)}$ refers to a diploid descendant of a diploid in the parental, and coming, except for $W_{0}^{(n)}$, from the cross of an $(n-1)$ th generation diploid with his haploid offspring. Odd $n$ refer to lines starting with a haploid and, for them,

$$
W_{i}^{(2 k+1)}=W_{i}^{(2 k)} .
$$

Acknowledgments.-The work has been financed by the Association Euratom/CNR/ CNEN, contract No. 012-61-12 BIAI. I am also indebted to Dr W. Matousek, Professor L. L. Cavalli-Sforza and Dr C. Matessi for their critical reading of the manuscript.

\title{
REFERENCE
}

KeMPthorne, o. 1957. An Introduction to Genetic Statistics, pp. $545+$ xvii. J. Wiley and Sons, New York.

\section{EFFECT OF FRACTIONATED DOSES OF RADIATION ON RECOMBINATION}

\author{
C. W. LAWRENCE
}

Wantoge Research Laboratory, (AERE), Berks, U.K.

Received 21.vii.67

Low, virtually non-lethal doses of ${ }^{60} \mathrm{Co}$ gamma radiation change gene recombination in Chlamydomonas reinhardi, and also chiasma frequency in Lilium longiflorum and Tradescantia paludosa, only if given during two short meiotic stages (Lawrence, 1965a, 1961 $a, b$ ). Preleptotene irradiation decreases the frequency of recombination while treatment in late zygotene or pachytene gives an increase. The response at both stages may be due to 
interference in the synthesis of DNA (Hastings, 1965; Davies and Lawrence, 1967) and it appears likely that processes leading to recombination occur in normal, unirradiated cells during, or close to, these responsive periods. A puzzling feature of this scheme is that the preleptotene events, unlike those at stage two, are far removed in time from the stage at which crossing-over takes place. The process of crossing-over may itself occur in two steps (Lawrence, 1965a, 1967a, b) or irradiation at stage one may interfere with chromosome pairing (Davies and Lawrence, 1967). The finding that the frequency of allelic recombination can be increased by irradiation during a short stage which is probably identical to that in which the frequency of gene recombination is decreased (Lawrence, 1967a), does not appear to support this "pairing" hypothesis. It is difficult to see how allelic recombination can occur in the absence of chromosome pairing. Nevertheless, such evidence is not conclusive and the experiment described here was designed to examine the "pairing" hypothesis more directly.

If irradiation at stage one inhibits chromosome pairing, the increase in recombination frequency expected from irradiation at stage two will be abolished or at least diminished by prior irradiation with a suitable dose at stage one. If, therefore, the extent of the increase is not diminished, an effect on chromosome pairing can be ruled out. The opposite result does not prove the existence of an effect on pairing, however, since other causes may give rise to it.

Meiosis in $C$. reinhardi occurs during the germination of the uninucleate zygospore, which can be synchronised (Levine and Ebersold, 1958). Grown under conditions described previously (Lawrence, 1965b; Lawrence and Davies, 1967), zygospores of the constitution arg $1+1+$ pab 2 used in this and earlier experiments consistently give a maximum response to radiation five and a half hours (stage 1), and six and a half hours (stage 2), after the start of germination. The success of the experiment hinges on the ability to irradiate any given zygospore during its sensitive period at both stage one and stage two. Each zygospore remains competent to respond to irradiation for only about 10-15 minutes at stage one and probably a similar period at stage two (Lawrence, 1965b). The standard deviation between zygospores for the time of entry into these responding stages is, however, about 30 minutes (Lawrence, loc. cit.). This means that the proportion of zygospores competent to respond at any one instant is, even at maximum, small. To ensure that a high proportion of zygospores were irradiated while competent and also that the majority of zygospores responding to treatment at stage one were re-irradiated while competent at stage two, irradiation at each stage was given in the form of six equal dose increments separated by ten-minute intervals. Low dose rate/long duration treatments, at first sight a more obvious solution to the problem, cannot be used since no response is obtained with low dose rate treatments at stage two (Lawrence, 1965b). The first increment at stage one was given 5 hours 5 minutes after the start of germination and at stage two the first increment was given at 6 hours 5 minutes. Zygospores irradiated during both stage one and stage two received twelve dose increments. In one series each increment was 750 rads and in another 1000 rads, delivered in air at a dose rate of $14.65 \mathrm{Kr} /$ minute. A single acute dose of 1000 rads gives about a half of the maximum response to acute doses at both stage one and stage two, and 750 rads gives about one-third of the maximum response (Lawrence, 1967b). Germination rates are unaffected 
by irradiation at these stages with doses at least as high as $35 \mathrm{Kr}$ (Davies, 1966).

The results of the experiment are shown in the table. The data demonstrate quite clearly that the capacity to respond to irradiation at stage two is not diminished by prior irradiation at stage one. In fact, this capacity

TABLE

Number of colonies counted on non-selective and selective media, and the proportion of zygospores giving rise to recombinant products (recombinant zygospores) following irradiation at stage one, stage two, or both stage one and stage two. The data are the pooled results of two experiments. In each experiment, ten pairs of petri dishes were counted in each treatment group in the 750-rad series, eight pairs in the 1000-rad series, and six pairs in the control

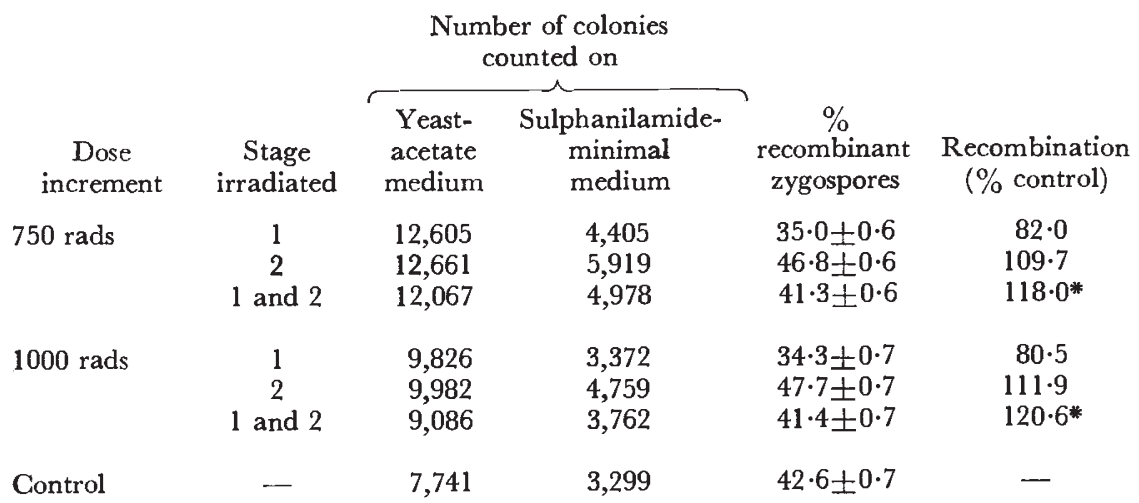

* Percentage of the frequency following irradiation at stage one.

appears to have been doubled, but the difference may merely reflect the difficulty of inferring changes in the frequency of crossing-over from recombination data, especially since the selective technique employed does not distinguish between single and certain multiple recombinant tetrads. The results appear, therefore, to rule out a radiation-induced inhibition of pairing at stage one.

The size of the response to irradiation at stage one with 750-rad dose increments is in good agreement with the expected response calculated in the manner described previously (Lawrence, 1965b). At stage two the agreement is less good, but this may arise from difficulties in determining the expected size of the response (Lawrence, loc. cit.). Disagreement between the sizes of observed and expected responses is more serious in the results obtained with the 1000-rad dose increments. The reason for the disagreement is unknown, but it seems unlikely that if known it would invalidate the main conclusion from the experiment. If irradiation at stage one does not inhibit chromosome pairing, the experiment provides indirect support for the two-step model of crossing-over suggested previously (Lawrence, 1967a,b). According to this scheme structural changes occur in chromosomal DNA during its premeiotic synthesis, possibly because the DNA is more vulnerable to such change at this stage. These regions of structural change, which occur in normal unirradiated cells, are potential sites for both crossing-over and gene conversion. Interaction between these sites during pachytene, involving their repair or correction, may lead to 
crossing-over. Alternatively, a single site on only one of the two non-sister chromatids may initiate crossing-over. Models to explain crossing-over in terms of repair or error correction have been proposed by Whitehouse (1963) and by Holliday (1964) and events at stage two may well follow one or other of these schemes.

\title{
SUMMARY
}

1. A comparison between the recombination frequencies in zygospores irradiated during both stage one (preleptotene) and stage two (late zygotenepachytene) and those irradiated in stage one only, suggests that irradiation at stage one does not inhibit chromosome pairing.

2. The results provide indirect support for the two-step model of crossingover (Lawrence, 1967a, b).

Acknowledgment.-I wish to thank Mr D. Cooke for his expert assistance.

\section{REFERENCES}

DAVIES, D. R. 1966. Patterns and rates of recovery in synchronous populations of algal cells exposed to gamma radiation. Rad. Res., 29, 222-235.

DAVIES, D. R., AND LAWRENCE, c. W. 1967. The mechanism of recombination in Chlamydomonas reinhardi. II. The influence of inhibitors of DNA synthesis on intergenic recombination. Mut. Res., 4, 147-154.

HASTINGS, P. J. 1965. Genetic recombination studies with Chlamydomonas reinhardi. Ph.D. Thesis, University of Cambridge.

HOllidAY, R. 1964. A mechanism for gene conversion in fungi. Genet. Res., 5, 282-304.

LAWRENCE, C. W. 1961a. The effect of the irradiation of different stages in microsporogenesis on chiasma frequency. Heredity, 16, 83-89.

LAWRENCE, C. $w$. 1961 b. The effect of radiation on chiasma formation in Tradescantia. Rad. Bot., 1, 92-96.

LAWRENCE, C. W. 1965a. Influence of non-lethal doses of radiation on recombination in Chlamydomonas reinhardi. Nature, 206, 789-791.

LAWRENCE, c. $w$. 1965 b. The effect of dose duration in the influence of irradiation on recombination in Chlamydomonas. Mut. Res., 2, 487-493.

LAWRENCE, C. W. 1967a. Influence of non-lethal doses of radiation on allelic recombination in Chlamydomonas reinhardi. Genet. Res., 9, 123-127.

LAWRENCE, c. W. 1967b. Radiation effects on genetic recombination in Chlamydomonas reinhardi. In The Effects of Ionising Radiations on Meiotic Systems. I.A.E.A., Vienna. (In press.)

LAWRENCE, C. W., AND DAvies, D. R. 1967. The mechanism of recombination in Chlamydomonas reinhardi. I. The influence of inhibitors of protein synthesis on intergenic recombination. Mut. Res., 4, 137-146.

IEVINE, R. P., AND EBERSOLD, W. T. 1958. Gene recombination in Chlamydomonas reinhardi. Cold Spring Harbour Symp. Quant. Biol., 23, 101-109.

WHITEHOUSE, H. L. K. 1963. A theory of crossing-over by means of hybrid deoxyribonucleic acid. Nature, 199, 1034-1040.

\section{SPLIT AND ENLARGED SATELLITES IN MAN}

\author{
S. P. RAY-CHAUDHURI, S. KAKATI and T. SHARMA \\ Department of Zoology, Banaras Hindu University, Varanasi-5, India
}

\section{INTRODUGTION}

All the five pairs of acrocentric chromosomes in man are satellited (Ferguson-Smith and Handmaker, 1961; German, 1964). Peculiarities in the size and structure of satellites have been reviewed by De La Chapelle 